\title{
Úlcera corneal en un caballo de silla argentino de Córdoba, Colombia
}

\author{
Corneal ulcer in an argentine chair horse of Córdoba, Colombia
}

\author{
Buitrago M, Jhonny ${ }^{1}$ M.Sc; Montes V, Donicer ${ }^{2}$ Ph.D, Cardona A, José ${ }^{*}$ Ph.D. \\ ${ }^{1}$ Universidad de Córdoba, Facultad de Medicina Veterinaria y Zootecnia, \\ Grupo de Investigación en Medicina de Grandes Animales (MEGA), Montería, Colombia. \\ 2Universidad de Sucre. Facultad de Zootecnia. \\ Departamento de Ciencias pecuarias. Sincelejo, Colombia.
}

\section{KEYWORDS:}

Eyes;

blepharitis;

ophthalmology;

cornea;

equine.

\section{ABSTRACT}

We report a case of an Argentine castrated male horse, about 8 years of age, which was attended by the outpatient clinic of the Medical-Surgical Clinic of Large Animals of the Faculty of Veterinary Medicine and Zootechnics of the University of Cordoba. The anamnesis indicates that the horse was in field work in a wooded land, which presented the next day of work increase in the production of tears and edema in the eyelids of the right eye, which was treated with eye drops antibiotic for 3 days. Fifteen days after the treatment was applied, the horse managers did not notice improvement, so they requested Veterinary Medical help. In the clinical evaluation of the animal, epifhora with purulent secretion, chemosis, conjunctivitis and edema with marked lesion of the corneal epithelium were found in the right eye. Special tests such as the Schrimer test were performed, with marked increase in lacrimal secretion; Subsequently the Fluorescein Sodium test was performed, evidencing the marked ulceration of the cornea. In definitive diagnosis, based on the special ophthalmic examination and the tests performed was of corneal ulcer.

\section{RESUMEN}

Se reporta un caso de un caballo de silla argentino, macho castrado, de 8 años de edad aproximadamente, el cual fue atendido por el servicio clínico ambulatorio del área de Clínica Médico-Quirúrgica de Grandes Animales de la Facultad de Medicina Veterinaria y Zootecnia de la Universidad de Córdoba. La anamnesis indica que el caballo se encontraba en labores de trabajo de campo en un terreno boscoso, que presento al siguiente día de trabajo aumento en la producción de lágrimas y edema en los párpados del ojo derecho, por lo que le fue aplicado tratamiento con colirio antibiótico por 3 días. Quince días después de aplicado el tratamiento, los encargados del caballo no notaron mejoría, por lo que solicitaron ayuda Médico Veterinaria. En la evaluación Clínica del animal, se encontró en el ojo derecho epifora con secreción purulenta, quemosis, conjuntivitis y edema con marcada lesión del epitelio corneal. Se realizaron pruebas especiales como el test de Schrimer, encontrándose aumento marcado en la secreción lagrimal; posteriormente se realizó la prueba de la Fluoresceína Sódica, evidenciando la marcada ulceración de la córnea. En diagnóstico definitivo, basado en el examen oftalmológico especial y las pruebas realizadas fue de úlcera corneal. 


\section{INTRODUCCIÓN}

En caballos las enfermedades oculares aunque comunes, frecuentemente no son evidentes a menos que se realice un examen oftalmológico especifico (HUGHES, 2010; BAUER, 2015). Pudiendo tener diversas etiologías etiologías, que comprometen la calidad de la visión dependiendo del grado de la afección y del compromiso de las estructuras oculares afectadas (CUNHA et al. 2015).

THANGADURAI et al. (2010) y BAUER, (2015), indícan la importancia del examen oftálmico especializado y sistemático, precedido de una buena historia clínica y de un adecuado examen físico general, para obtener un diagnóstico temprano y acertado de los trastornos oculares. De igual forma BAUER, (2015), expresa que entre más rápido es diagnosticada una enfermedad oftálmica mejor será su respuesta al tratamiento y su pronóstico.

La terapia indicada y el tiempo de recuperación pueden variar también con factores como el agente etiológico, la estructura afectada y la gravedad de la lesión (CUNHA et al. 2015). Por lo que HUGHES, (2010), manifiesta que el reconocimiento de las manifestaciones oculares de enfermedades sistémicas puede permitir dirigir el tratamiento oftálmico con beneficios potenciales para limitar la patología ocular y perdida de la visión. Así mismo BAUER, (2015), informa que en los casos en los cuales la enfermedad ocular resulta en ceguera o en un ojo doloroso se requerirá de enucleación, siendo esencial la remisión del globo ocular para evaluación microscópica en la determinación de la causa de la ceguera, permitiendo así tener un pronóstico para el ojo contralateral.

El estudio de las alteraciones oftalmológicas de los equinos se ha centrado en alteraciones particulares como la uveítis recurrente equina, las neoplasias oculares y las ulceras corneales (BROOKS, 2012; BROOKS, 2014a; CARVALHO et al. 2014; MONTGOMERY, 2014; WITKOWSKI et al. 2016) y los estudios de prevalencia se han realizado principalmente mediante el análisis de la casuística clínica de hospitales (HURN \& TRUNER, 2006; THANGADURAI et al. 2010; TAMILMAHAN et al. 2013).

En el presente informe se reporta una de las patologías oculares más comunes en equinos (MONTES et al. 2016), tratándose de una ulceración corneal en un caballo de silla argentino atendido por el Servicio Clínico Ambulatorio de Grandes Animales de la Facultad de Medicina Veterinaria de la Universidad de Córdoba en el municipio de Montería, Córdoba.

\section{MATERIALES Y MÉTODOS}

Un caballo de Silla Argentino, de 8 años de edad y un peso aproximado de $550 \mathrm{Kg}$. Fue atendido por el servicio clínico ambulatorio del área de Clínica MédicoQuirúrgica de Grandes Animales de la Facultad de Medicina Veterinaria y Zootecnia de la Universidad de Córdoba.

Entre los datos anamnesicos, se tiene que el caballo se encontraba en labores de trabajo de campo en un terreno boscoso. Al siguiente día de trabajo se detectó aumento en la producción de lágrimas y edema en los párpados del ojo derecho, por lo que le fue aplicado por 3 días seguidos un colirio por parte de los operarios. Quince días después, los encargados del caballo no notaron mejoría, por lo que solicitaron ayuda Médico Veterinaria por parte del servicio clínico ambulatorio de la Facultad de Medicina Veterinaria de la Universidad de Córdoba, por lo que fue solicitada la realización de pruebas especiales del área afectada.

En la evaluación Clínica del animal, se encontró en el ojo derecho epifora con secreción purulenta, quemosis, conjuntivitis y edema con marcada lesión del epitelio corneal (Figura 1). Se realizaron pruebas especiales como el test de Schrimer, encontrandose aumento marcado en la secreción lagrimal; posteriormente se realizó la prueba de la Fluoresceína Sódica, evidenciando la marcada ulceración de la córnea (Figura 2). En el resto de sus sistemas presentaba normofuncionalidad.

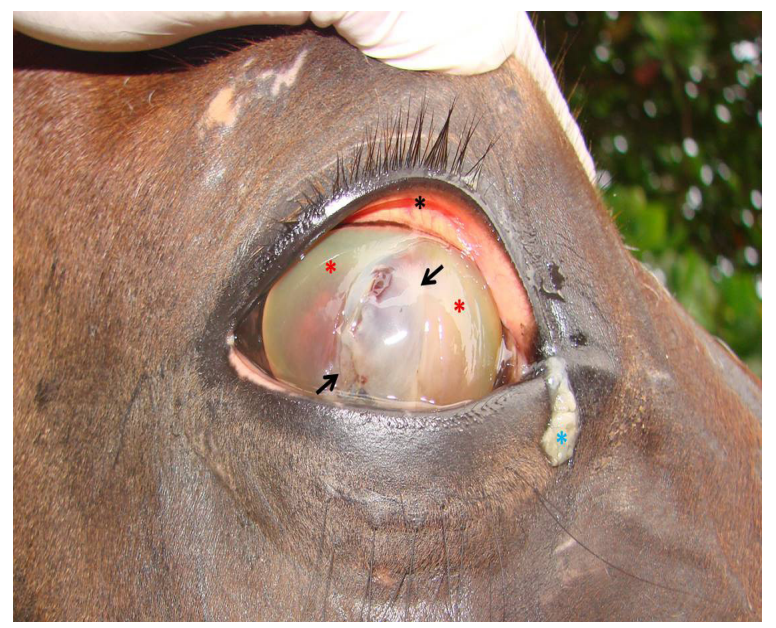

Figura 1. Úlcera corneal en un caballo. Observe la secrción purulenta en el canto medial (Asterisco azul), la conjuntivitis evidenciada por la coloración rojiza de la conjuntiva (Asterisco negro), el edema de la córnea (Asteriscos rojos), así como la solución de continuidad de la córnea (Flechas). 


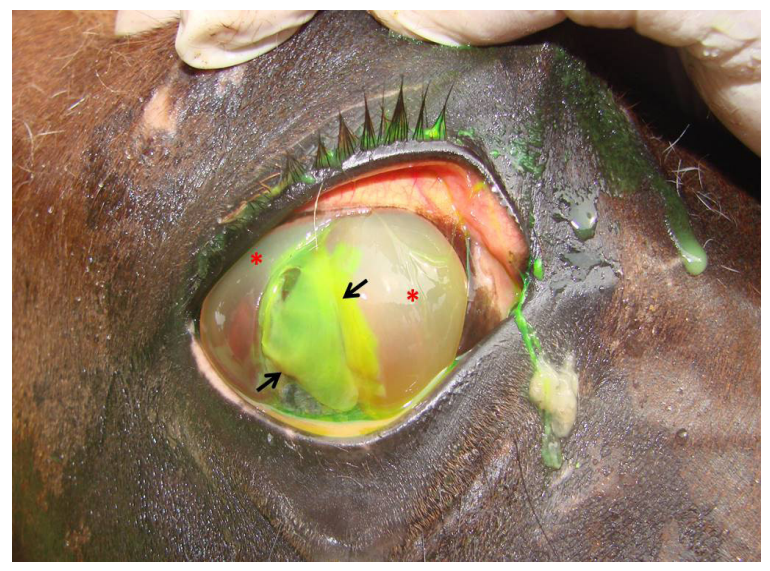

Figura 2. Prueba de la fluoresceína sódica en un caballo con úlcera corneal. Observe la el edema de la córnea (Asteriscos rojos), así como la solución de continuidad de la córnea coloreada de verde (Flechas).

\section{RESULTADOS Y DISCUSIÓN}

TAMILMAHAN et al. (2013) y MARTINS \& BARROS (2014), manifiestan que los equinos se encuentra dentro de las especies animales más susceptibles a padecer de alteraciones oculares; es así como HURN \& TRUNER (2006), informan que un $67,6 \%$ de los animales en su estudio presentó algún tipo de alteraciones oftalmológicas. Sin embargo, BAUER (2015) y MALALANA (2016) expresan que aunque las enfermedades oculares son comunes en el equino, solo el $3,3 \%$ de los propietarios reportan problemas oculares en sus caballos, siendo la descarga ocular el motivo de consulta mas comun, y solo $2,6 \%$ perciven la vision como un importante tema de salud, por lo que existe un gran numero de caballos que pueden presentar problemas oculares sin diagnosticar.

MONTES et al. (2016), reportaron una mayor afección en las estructuras oculares que en las peri oculares, con un predominio de las patologías corneales siendo las úlceras corneales la segunda patología ocular de mayor presentación en caballos de vaquería del departamento de Córdoba, seguida de las conjuntivitis y de las blefaritis respectivamente.

El caso clínico del presente estudio, presento evidencia clínica de alteración ocular, siendo medicado por personal no profesional, por lo que pudo ser progresivo el cuadro. Lo que concuerda con lo informado por REICHMAN et al. (2008) que dicen que si este tipo de enfermedades no son tratadas a tiempo y de forma adecuada pueden terminar en ceguera, por lo que la principal importancia de las enfermedades oftálmicas es el grado de compromiso visual que puede inutilizar a los animales para cumplir con el trabajo destinado. De igual forma THANGADURAl et al. (2010), indican que además puede tener efectos nocivos (deletéreos) tanto en los caballos como en sus jinetes, siendo necesario incluir el examen ocular como una parte del examen físico de rutina en la práctica equina.

El presento caso de queratitis ulcerativa, coincide con lo informado con MONTES et al. (2016) y difieren de los hallados en otros lugares del mundo, como india, que reportaron que la opacidad corneal con mayor frecuencia (TAMILMAHAN et al. 2013), en Australia las alteraciones de la retina y el cristalino fueron las más observadas (HURN \& TRUNER , 2006). En Brasil encontraron en équidos de tracción las alteraciones corneales (REICHMAN et al. 2008). CUNHA et al. (2015), reportaron en Brasil que el mayor motivo de consulta oftalmológica correspondían a ulceras corneales; mientras que LABELLE (2011), la encontro como la tercera causa de alteraciones oculares.

La cornea fue la estructura ocular afectada en el presente estudio, lo que concuerda con lo reportado por BROOKS et al. (2014b), quienes informaron las patologías corneales con la mayor cantidad de diagnósticos en su estudio, informando además, que las queratopatias pueden ser ulcerativas o no ulcerativas, infecciosas o estériles.

Clínicamente las queratitis ulcerativas son la enfermedad oftálmica más común del caballo, que al ser una enfermedad amenazante de la visión requiere un diagnóstico clínico temprano y una apropiada terapia medica siendo el principal (BROOKS, 2012; BROOKS, 2014a; KIRK, 2014). La queratitis ulcerativa puede presentarse inicialmente como una ulcera epitelial menor o infiltrados celulares con dolor leve, blefaroespasmo epifora y fotofobia, la leve perdida de pestañas del parpado superior puede ser un signo temprano y sutil de ulceración corneal. Al inicio la uveítis anterior y la vascularización corneal pueden no ser clínicamente pronunciadas. El diagnostico se realiza mediante la retención de fluoresceína. (BROOKS, 2014b).

Las ulceras corneales pueden ir de simples rupturas superficiales, abrasiones del epitelio corneal, derretimiento del colágeno corneal, hasta perforación corneal de espesor completo con prolapso de iris, siendo posibles secuelas la ruptura del globo ocular, Phthisis bulbi y ceguera. El ojo prominente del caballo puede predisponer a ulcera corneal de tipo traumático (BROOKS, 2012; BROOKS, 2014b). En este estudio las principales alteraciones corneales correspondieron a la queratitis no ulcerativa, queratitis ulcerativa y la queratitis superficial crónica (Pannus).

En conclusión las alteraciones del sistema oftálmico son comunes en los caballos, por lo que existe una predisposición a este tipo de lesiones en caballos de trabajo. Se hace necesario estudios a nivel poblacional para conocer la prevalencia de las alteraciones oftálmicas en equinos del departamento de Córdoba, así como sus factores de riesgo para poder realizar planes de prevención y control. 


\section{REFERENCIAS}

BAUER, B. 2015. Ocular pathology. Vet Clin Equine, 31, 425-448.

BROOKS, D. 2012. Equine Corneal Ulceration. Proceedings of the AAEP Focus on Opthalmology, (págs. 1-11). Raleigh, NC, USA.

BROOKS, D. 2014a. How to Reach the Medical Standards of Care for Ulcerative and Non-Ulcerative Equine Keratopathies. AAEP PROCEEDINGS, 60, 11-15.

BROOKS, D. 2014b. How to Use the Clinical Examination to Determine the Significance of Abnormalities of the Horse Cornea and Adnexa. AAEP PROCEEDINGS, 60, 19-24.

CARVALHO, F; DANTAS, A; RIET-CORREA, F; ANDRADE, R; NETO, P; NETO, E. 2014. Estudo retrospectivo das neoplasias em ruminantes e equídeos no semiárido do Nordeste Brasileiro. Pesq. Vet. Bras., 34(3), 211-216.

CUNHA, F; DA ROSA, B; SOARES, L; PAZINATO, F; SOARES, P; WYNE, C. 2015. Alterações do sistema oftálmico em equinos com ênfase em medidas terapêuticas. Acta Scientiae Veterinariae, 43(suplemento 1), 99-106.

HUGHES, K. 2010. Ocular manifestations of systemic disease in horses. EQUINE VETERINARY JOURNAL, 37, 89-96.

HURN, S; TRUNER, A. 2006. Ophthalmic examination findings of thoroughbred racehorses in australia. veterinary ophthamology, 9(2), 95-100.

KIRK, N. 2014. Essentials of Veterinary Ophthalmology (tercera edicion ed.). Gainesville, Florida, USA: Wiley blackwell.

MALALANA, F. 2016. Ophthalmologic Disorders in Aged Horses. Vet Clin Equine, 32, 249-261.

MARTINS, T; BARROS, C. 2014. Fifty years in the blink of an eye: a retrospective study of ocular and periocular lesions in domestic animals. Pesq. Vet. Bras, 34(12), 1215-1222.

MONTES, D; BUITRAGO, J; CARDONA, J. 2016. Frecuencia de patologías oculares en caballos de vaquería en explotaciones ganaderas del departamento de Córdoba, Colombia. Rev Colombiana Cienc Anim, 8(Supl):377-385.

MONTGOMERY, K. 2014. Equine ocular neoplasia: A review. Equine Veterinary Education, 26(7), 372-380.

LABELLE, A. 2011. Ophthalmic lesions in neonatal foals evaluated for nonophthalmic disease at referral hospitals. JAVMA, 239(4), 486-492.

REICHMAN, P; DE OLIVEIRA, A; CARONATO, T. 2008. Ocurrence of ophthamologic diseases in horses used for urban cart hauling in londrina, PR, Brazil. Ciencia Rural Santa Maria, 38(9), 2525-2528.

TAMILMAHAN, P; ZAMA, M; PATHAK, R; MUNEESWARAN, N; KARTHIK, K. 2013. A retrospective study of ocular occurrence in domestic animals: 799 cases. Veterinary World, 6(5), 274-276.

THANGADURAI, R; SHARMA, S; BALI, D; MAHAJAN, V; SAMANTA, I; HAZRA, S. 2010. Prevalence of ocular disorders in an indian population of horses. journal of equine veterinary science, 30(6), 326-329.

WITKOWSKI, L; CYWANSKA, A; PASCHALIS-TRELA, K; CRISMAN, M; KITA, J. 2016. Multiple etiologies of equine recurrent uveitis - A natural model for human autoinmune uveitis:a brief review. comparative inmunology, microbiology and infectious diseases, 44, 14-20. 